Pacific Journal of Mathematic 


\title{
CONTRIBUTIONS TO THE THEORY OF DIVERGENT SERIES
}

\author{
I. HELLER
}

\section{INTRODUCTION}

We shall be concerned throughout with methods of summability consistent with the method of analytic continuation $(P)$. We recall that two methods are called consistent if whenever they are simultaneously effective they yield identical generalized sums. This property is not a consequence of regularity.

The purpose of this paper is to examine in a general way the class of methods consistent with $(P)$ and subject to the following agreements.

1. For greater generality, regularity of the methods will not be assumed.

2. The class shall be closed with respect to multiplication; that is, the result of successive application of two methods of the class shall represent a method of the class. To this end consistency is postulated, not only for the case in which the series is summed, but in a general way (see postulates below).

We are thus led to consider the class of matrix transformations defined by the following postulates.

(I) Any series $\sum u_{n}$, summable $(P)$, is transformed into a series $\sum v_{k}$, summable $(P)$

(II) $\sum_{u_{n}}(P)=\sum_{v_{k}}(P)$.

The class defined by these conditions is the exact analogue of the class of regular matrices, which may be defined in a similar fashion if "summable $(P)$ " and " $(P)$ " in (I) and (II) are replaced by "convergent" and "convergence", respectively.

\section{The CLass $\mathfrak{S}_{a a}$}

1.1. Notations and Definitions. The partial sums of a series $\sum_{u_{n}}$ will be denoted by $U_{n}$ : 


$$
U_{n}=u_{0}+u_{1}+u_{2}+\cdots+u_{n} \text {. }
$$

Also for series of variable terms:

$$
U_{n}(z)=u_{0}(z)+u_{1}(z)+\cdots+u_{n}(z) .
$$

The matrix of a series-to-series transformation will be denoted by $C=$ $\left(c_{k n}\right)$, where generally $k, n=0,1,2, \ldots$; only in Theorem 1 and its proof we suppose $k, n=1,2, \cdots$.

By $\sum_{c_{k n} u_{n}=v_{k}}$ the series $\sum_{u_{n}}$ is transformed into the series $\sum_{v_{k}}$.

The matrix of a sequence-to-sequence transformation will be denoted by $A=\left(a_{k n}\right)$. By $V_{k}=\sum a_{k n} U_{n}$, the sequence $\left(U_{n}\right)$ is transformed into the sequence $\left(V_{k}\right)$.

If $\left|u_{n}\right| \leq M^{n+1}(n=0,1, \cdots)$, then $\sum_{u_{n}}$ will be said to be an analytic series (as then $\sum_{u_{n} z^{n}}$ determines an analytic function $f(z)$, regular at the origin).

By $\mathbb{C}_{a a}$ we denote the class of matrices $C$ which transform any analytic series into an analytic series, while by $\mathfrak{S}_{a a}^{\prime}$ we denote the class of matrices $A$ which transform the sequence of partial sums of any analytic series into the sequence of partial sums of an analytic series.

1.2. Theorems. Let $C=\left(c_{k n}\right)$ be a matrix, where $k, n=1,2, \cdots$.

THEOREM 1. In order that $C$ should belong to $\mathfrak{S}_{a a}$, that is to say in order that $C$ should transform each analytic series into an analytic series, it is necessary and sufficient that to each $\epsilon>0$ there exists an $M_{\epsilon}>0$ such that

$$
\left|C_{k n}\right| \leq \epsilon^{n} M_{\epsilon}^{k}
$$

$$
(k, n=1,2, \cdots) \text {. }
$$

CoROLlaRY 1. If $C$ belongs to $\mathfrak{S}_{a a}, \sum_{u_{n}}$ is analytic, and $\sum\left|c_{k n} u_{n}\right|=$ $w_{k}$, then $\sum w_{k}$ is analytic.

COROLLARY 2. If $C$ is a triangular matrix, that is to say, if

$$
c_{k n}=0 \text { when } n>k,
$$

then condition (1.2.1) is equivalent to either of the conditions:

$$
\left|c_{k n}\right| \leq P^{k}
$$

$$
N_{k} \leq R^{k}, \text { where } N_{k}=\sum\left|c_{k n}\right|
$$


THEOREM 2. The class $\mathfrak{S}_{a a}^{\prime}$ is identical with $\mathfrak{\Im}_{a a}$; that is to say: in order that A should transform the sequence of partial sums of each analytic series into the sequence of partial sums of an analytic series, it is necessary and sufficient that to each $\epsilon>0$ there exists an $M_{\epsilon}>0$ such that

$$
\left|a_{k n}\right| \leq \epsilon^{n} M_{\epsilon}^{k}
$$

$(k, n=1,2, \cdots)$.

REMARK. If the indices $k, n$ of the matrix begin with 0 , then conditions (1.2.1)-(1.2.4) evidently run:

$$
\begin{aligned}
& \left|c_{k n}\right| \leq \epsilon^{n+1} M_{\epsilon}^{k+1}, \\
& \left|c_{k n}\right| \leq P^{k+1}, \\
& N_{k} \leq R^{k+1}, \quad(k, n=0,1,2, \cdots) .
\end{aligned}
$$

We first prove the corollaries and Theorem 2.

Proof of Corollary 1. The result is evident.

Proof of Corollary 2. Condition (1.2.1) implies (1.2.3) for any arbitrary matrix; indeed, for $\epsilon<1$ and

$$
R=\frac{M_{\epsilon}}{1-\epsilon}
$$

we obtain

$$
N_{k}=\sum\left|c_{k n}\right| \leq M_{\epsilon}^{k} \sum \epsilon^{n}=\frac{\epsilon M_{\epsilon}^{k}}{1-\epsilon} \leq\left(\frac{M_{\epsilon}}{1-\epsilon}\right)^{k}=R^{k} \quad(k=1,2, \cdots) .
$$

Further, it is plain that (1.2.3) implies (1.2.2). Finally the chain is closed by the proof that (1.2.2) implies (1.2.1): for $\epsilon \geq 1$ there is nothing to prove; for $\epsilon<1$ we put $M_{\epsilon}=P / \epsilon$ and obtain

$$
\left|c_{k n}\right| \leq P^{k}=\epsilon^{k} M_{\epsilon}^{k} \leq \epsilon^{n} M_{\epsilon}^{k} \quad(k=1,2, \cdots),
$$

the last relation holding because of $n \leq k$.

REMARK. In the general case, the condition of the theorem is not equivalent 
to those of Corollary 2, which continue to be necessary, but not sufficient, as shown by the following example:

Take as the $k$ th row of $C$ the coefficients of the Taylor development of the function

$$
f_{k}(z)=e^{z^{k}}
$$

the matrix $C$ thus defined satisfies (1.2.3), because of

$$
N_{k}=\sum\left|c_{k n}\right|=f_{k}(1)=e ;
$$

but the series $u_{n}=r^{n}$ is transformed into $v_{k}=e^{r^{k}}$, which, for $r>1$, is not analytic, since

$$
\left(e^{r^{k}}\right)^{1 / k}=e^{r^{k / k}} \longrightarrow \infty \text { as } k \rightarrow \infty \text {. }
$$

It will be observed that Theorem 1 is quite analogous to the theorem of Toeplitz-Schur, which establishes " $N_{k}$ bounded" as a necessary and sufficient condition in order that $C$ should transform every bounded sequence into a bounded sequence. This analogy persists also in the proof of Theorem 1 (see 1.3).

Proof of Theorem 2. If $f(z)=\sum_{u_{n}} z^{n}$ is convergent in a circle of radius $R$, and $g(z)=\sum z^{n}$, then the Cauchy product

$$
f(z) \cdot g(z)=h(z)=\sum U_{n} z^{n}
$$

is convergent in a circle of radius $r=\operatorname{minimum}(R, 1)$.

Conversely, if $h(z)=\sum U_{n} z^{n}$ converges in a circle of radius $r$, then $h(z) / g(z)=f(z)=\sum u_{n} z^{n}$ converges in the same circle.

This signifies that either the series $\sum u_{n}$ and $\sum U_{n}$ are both analytic, or neither of them is analytic. The series $\sum v_{k}$ and $\sum V_{k}$ behave in the same way. Hence, to say that $A$ transforms the partial sums of each analytic series $\sum_{u_{n}}$ into the partial sums of an analytic series $\sum_{v_{k}}$, is the same as to say that $A$ transforms each analytic series $\sum U_{n}$ into an analytic series $\sum V_{k}$.

1.3. Proof of Theorem 1. a) The condition is sufficient: If $\left|u_{n}\right| \leq M^{n}$, then, for $\epsilon<M^{-1}$, which implies $\epsilon M=q<1$, we obtain

$$
\left|v_{k}\right|=\left|\sum_{c_{k n}} u_{n}\right| \leq \sum\left|c_{k n} u_{n}\right| \leq \sum \epsilon^{n} M_{\epsilon}^{k} M^{n}=M_{\epsilon}^{k} \sum(\epsilon M)^{n}
$$




$$
=\frac{q}{1-q} \cdot M_{\epsilon}^{k} \leq\left(\frac{1}{1-q} \cdot M_{\epsilon}\right)^{k}=R^{k} \quad(k \geq 1) .
$$

b) The condition is necessary: We first remark that in order for $C$ to be applicable to each analytic series, the rows of $C$ must necessarily represent integral functions, that is,

$$
\sum_{c_{k n}} z^{n} \text { must converge for every } z \text {. }
$$

Secondly, the columns of the unit matrix must be transformed into analytic series; this signifies that each column of $C$ is analytic:

$$
\left|c_{k n}\right| \leq M_{n}^{k}
$$

Now the proof follows indirectly. From the hypothesis that $C$ belongs to $\mathfrak{S}_{a a}$ without satisfying (1.2.1), a contradiction is derived by constructing a certain analytic series, the transform of which is not analytic.

If (1.2.1) is not satisfied, then for a certain $\epsilon$ there exists no $M$ such that (1.2.1) holds; for this $\epsilon$ and any $M$ there exists a nonempty set $E_{M}$ of the $c_{k n}$, such that

$$
\left|c_{k n}\right|>\epsilon^{n} M^{k} \text { for all } c_{k n} \text { of } E_{M} \text {. }
$$

$E_{M}$ may be supposed infinite.

By variation of $M>0$ we obtain a family of sets $E_{M}$ such that $E_{M} \subseteq E_{M^{*}}$ when $M \geq M^{*}$.

We now remark:

(a) A row of $C$ can contain only a finite number of elements of $E_{M}$; if it contained an infinite number of elements, we would have, for that row, because of (1.3.3),

$$
\lim \sup \left|c_{k n}\right|^{1 / n} \geq \epsilon,
$$

in contradiction to (1.3.1).

(b) Given a fixed column (or a finite number of columns) of $C$, there exists an $M_{s}$ such that, for $M \geq M_{s}, E_{M}$ contains no element of this column (these columns ). In fact (1.3.2) implies

$$
\left|c_{k n}\right| \leq M_{n}^{k}=\epsilon^{n} \cdot \frac{M_{n}^{k}}{\epsilon^{n}} \leq \epsilon^{n}\left(\frac{M_{n}}{\epsilon^{n}}\right)^{k} ;
$$


hence, for $M_{s}=M_{n} / \epsilon^{n}$, we have $\left|c_{k n}\right| \geq \epsilon^{n} M_{s}^{k}$, (Here we have supposed that $\epsilon<1$; if $\epsilon \geq 1$, we set $M_{s}=M_{n}$ ).

After these preparations there can be constructed an analytic series, the transform of which is not analytic.

To that effect we first define, by induction, three increasing sequences of positive integers

$$
\left(M_{i}\right),\left(k_{i}\right),\left(n_{i}\right)
$$

For the index 1 the three numbers are chosen arbitrarily. Supposing the sequences constructed up to the index $\mathrm{i}-1$, we define the terms belonging to the index $\mathrm{i}$ as follows:

1. $M_{i} \geq M_{i-1}+1$, and [Remark (b)] such that

$$
\left|c_{k n}\right| \leq \epsilon^{n} M_{i}^{k} \text { for } n<n_{i-1} \text { and every } k \text {; }
$$

2. $M_{i}^{\prime}=\left(1+n_{i-1}\right) M_{i}+1$;

3. $k_{i}=$ the first index $>k_{i-1}$, such that the relation

$$
\left|c_{k_{i} n}\right|>\epsilon^{n} \cdot M_{i}^{k_{i}}
$$

$$
\text { holds for some } n \quad\left(\text { certainly } \geq n_{i-1}\right) \text {; }
$$

4. $n_{i}^{\prime}$ is such that $\left|c_{k_{i} n}\right| \leq \epsilon^{n} M_{i}^{\prime k_{i}}$ for $n \geq n_{i}^{\prime} \quad$ [Remark (a)];

5. $n_{i} \geq n_{i}^{\prime}$ and such that

$$
\sum_{n=n_{i}}^{\infty}\left|c_{k_{i} n}\right|<1 \quad \text { (the rows being convergent). }
$$

Setting now, for $0 \leq n<n_{1}$,

$$
u_{n}=0 \text {, }
$$

and, for $n_{i-1} \leq n<n_{i}$,

$$
u_{n}= \begin{cases}\frac{\left|c_{k_{i} n}\right|}{\epsilon^{n} \cdot c_{k_{i} n}} & \text { when } c_{k_{i} n} \neq 0, \\ 0 & \text { when } c_{k_{i} n}=0,\end{cases}
$$


we obtain, for $i>1$,

$$
\begin{aligned}
\left|v_{k_{i}}\right|=\left|\sum c_{k_{i} n} u_{n}\right| & \geq \sum_{n=n_{i-1}}^{n_{i}-1} c_{k_{i} n} u_{n}-\sum_{n=0}^{n_{i-1}-1}\left|c_{k_{i} n} u_{n}\right|-\sum_{n=n_{i}}^{\infty}\left|c_{k_{i} n} u_{n}\right| \\
& >M_{i}^{k_{i}}-n_{i-1} \cdot M_{i}^{k_{i}}-1>M_{i}^{k_{i}} ;
\end{aligned}
$$

thus

$$
\left|v_{k_{i}}\right|>M_{i}^{k_{i}}
$$

The series $\left(u_{n}\right)$, which is certainly analytic since

$$
\left|u_{n}\right|=\frac{1}{\epsilon^{n}} \text { or } 0
$$

is transformed into the series $\left(v_{k}\right)$, of which a partial sequence $\left(v_{k_{i}}\right)$ is miorized by $\left(M_{i}^{k_{i}}\right)$.

The sequence $\left(M_{i}\right)$ tends to infinity because of

$$
M_{i} \geq M_{i-1}+1
$$

therefore $\lim \sup \left|v_{k}\right|^{1 / k}=\infty$, which implies that $\left(v_{k}\right)$ is not analytic, in contradiction to the hypothesis. This completes the proof of Theorem 1 .

1.4. Corresponding transformations. The product of two matrices of $\mathfrak{S}_{a a}$ exists, and belongs to $\mathfrak{S}_{a a}$. The multiplication is associative:

$$
A(B C)=\left(\begin{array}{lll}
A B & B
\end{array}\right)=A B C .
$$

We have to show that

$$
\sum_{\lambda} \sum_{\nu} a_{k \lambda} b_{\lambda_{\nu}} c_{\nu n}=\sum_{\nu} \sum_{\lambda} a_{k \lambda} b_{\lambda \nu} c_{\nu n}
$$

We write $\sum_{\nu}\left|b_{\lambda_{\nu}} c_{\nu n}\right|=w_{\lambda_{n}}$.

By (1.3.2) and Corollary 1 of Theorem 1 , the sequence $w_{\lambda_{n}}$ ( $n$ fixed) is analytic. Hence

$$
\sum_{\lambda}\left|a_{k \lambda} w_{\lambda_{n}}\right|
$$


is convergent. This absolute convergence of the double series of (1.4.2) implies that the summations are invertible.

A sequence may be written as a matrix having the sequence as first column, while the other columns contain only zeros.

Evidently it means just the same to say that the sequence is analytic, or that the matrix belongs to $\mathfrak{F}_{a a}$.

The sequence of partial sums $U_{k}$ of a sequence $u_{n}$ is obtained in transforming $u_{n}$ by a matrix $B$,

$$
U_{k}=B\left(u_{n}\right) \text {, }
$$

where

$$
B=\left(b_{k n}\right), \quad b_{k n}=\left\{\begin{array}{lll}
0 & \text { when } n>k \\
1 & \text { when } n \leq k .
\end{array}\right.
$$

Evidently $B$ and its inverse $B^{-1}$ belong to $\mathcal{S}_{a a}$.

In $\mathfrak{S}_{a a}$ a matrix $A$, regarded as a sequence-to-sequence transformation, and a matrix $C$, regarded as a series-to-series transformation, may be said to be corresponding or associated when, for each analytic series, the $A$-transform of the partial sums is the sequence of partial sums of the $C$-transform of the series.

This correspondence is biunique. We demand indeed that

$$
\sum c_{k n} u_{n}=v_{k}, \quad \sum a_{k n} U_{n}=V_{k} \text { and }\left(U_{k}\right)=B\left(u_{n}\right)
$$

should imply

$$
\left(V_{k}\right)=B\left(v_{k}\right) \text {, that is to say, } A B\left(u_{n}\right)=B C\left(u_{n}\right) \text {, }
$$

for each analytic series $\sum u_{n}$.

This is equivalent to $A B=B C$, which yields

$$
A=B C B^{-1}, C=B^{-1} A B
$$

\section{The Classes $\mathbb{S}_{t t}$ aND $\mathbb{S}_{\overparen{H}}$}

2.1. Definitions of the Classes. If the method $(P)$ of analytic continuation is restrained to the open Mittag-Leffler star, then it will be denoted by $(P t)$. Thus an analytic series $\sum_{u_{n}}$ will be said to be summable $(P t)$, if the point $z=1$ is situated in the Mittag-Leffler star (briefly: star) of the principal branch of the analytic continuation of $f(z)=\sum_{u_{n}} z^{n}$. 
Definition of the class $\mathfrak{S}_{t t}$. $\mathfrak{S}_{t t}$ is defined as the class of matrices transforming every series that is summable $(P t)$, into a series summable $(P t)$ to the same $(P t)$ sum. They thus transform a point $b$ of the star of a function $F(z)$ into the point $y=1$, situated within the star of a function $H(y)$, such that $F(b)=$ $H(1)$.

We shall use the following notations:

$$
\begin{array}{ll}
\sum_{u_{n} z^{n}=f(z)} & \sum_{c_{k n} y^{k}=c_{n}(y)} \\
\sum_{c_{k n} u_{n} z^{n}=v_{k}(z)} & \sum_{v_{k}(z) y^{k}=f(z, y)}
\end{array}
$$

$F(z)$ : analytic continuation of $f(z)$ along straight lines through the origin [uniform principal branch, represented, for small $z$, by $f(z)$ ]. $F(z, y)$ : analytic continuation of $f(z, y)$, with regard to $y$ (uniform principal branch), for each fixed $z$.

For the geometric series we write $g$ and $G$ instead of $f$ and $F$; thus:

$$
g(z)=\sum z^{n}, \quad G(z)=1 /(1-z), \quad g(z, y), \quad G(z, y) .
$$

Under certain conditions the behaviour of a transformation when applied to the geometric series $\sum z^{n}$ admits conclusions about how it will behave in the general case of an arbitrary analytic series. This principle is used throughout the remainder of this paper. The conclusions on behaviour will pass from a given domain $\&$ of $G(z)$ to a certain domain $\mathcal{F}$ of $F(z)$, where $\mathcal{F}$ depends on $F$ and $G$.

Definition of $\widetilde{\xi}=\widetilde{F}(F$, अ $)$. We denote by

(8*: the star of $1 /(1-z)$ (that is, the whole $z$-plane except $[1, \infty]$ of the real axis);

(4) : an open connected domain containing $z=0$, and situated within $\mathbb{B}^{*}$; it is further supposed that for each $R>0$, the part of $\&$ situated in the circle $|z|<R$ has a rectifiable boundary;

$\tau^{*}$ : the star of $F(z)$;

$\subseteq$ : the border of $z^{*}$;

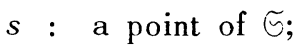

$F_{s}$ : the domain symbolically defined by $s$. \&, that is, the set of points obtained by multiplying every point of $\mathbb{E}$ by a fixed $s$ of $\Xi$. 
Then $₹$ is defined as the intersection of all $q_{s}$. We may write symbolically

$$
\mathfrak{\xi}(F, \otimes)=\Im\left(\widetilde{\mho}_{s}\right)=\mathfrak{\Im}(s \cdot \otimes) \quad(s \in \circlearrowleft) .
$$

REMARKS. l. If $\mathbb{E}$ is star-shaped, then $\mathfrak{F}$ does not change when $\subseteq$ is defined as consisting of all boundary points of $\mathcal{F}^{*}$ and an additional arbitrary set of points exterior to $\widetilde{f}^{*}$. Especially $\subseteq$ may be the part of the plane complementary to $\widetilde{r}^{*}$, or, in the case of a single-valued function, the set of all singular points.

Indeed, if $p$ is a point exterior to $飞^{*}$, then on the segment $O p$ there is a singular point $s$, and $p=r \cdot s$, where $r>1$. Hence

$$
\mho_{p}=p \cdot \mathbb{B}=r \cdot s \cdot \mathbb{B}=r \cdot \mho_{s} \cdot
$$

This implies that $\mathfrak{F}_{p}$ contains $\widetilde{F}_{s}$, and consequently $\mathfrak{F}_{p}$ has no influence on the intersection $\mathfrak{\Im}\left(\mathfrak{F}_{s}\right)$.

2. $\mathcal{F}$ is open and contains the origin (and thus is never empty). To see that it is open, we have to show that if $b$ belongs to $\mathcal{F}$ then so does a certain neighborhood of $b$. By definition of $\mathfrak{F}$, the set $b / \widetilde{G}$ is contained in $\mathbb{G}$. As $\widetilde{G}$ is closed, and does not contain the origin, $b / \subseteq$ is closed and bounded. Since $\&$ is open, there is a certain $r>0$ such that, for each $s$ of $\in$, the circle $K(b / s, r)$, of centre $b / s$ and radius $r$, is in $\mathbb{B}$. Then $\widetilde{F}_{s}=s$. \& contains in particular the set $s \cdot K(b / s, r)=K(b, r|s|)$. Now let $\sigma>0$ be such that $\sigma \leq|s|$ for each $s$ of $\mathbb{G}$. Then, for each $s$ of $\mathcal{G}, K(b, r \cdot \sigma)$ is contained in $\mathfrak{F}_{s}$, and consequently in $\mathfrak{F}$.

\section{Special cases:}

(a) If $\&$ is the circle $|z|<1$, then $₹$ is the circle of convergence of $F(z)$.

(b) If $\&$ is the half-plane $\Re(z)<1$, then $₹$ is the Borel polygon of $F(z)$.

(c) If $\&=B^{*}$, then $₹=\mho^{*}$.

(d) If $\subseteq$ is empty, then $\mathfrak{f}$ is the whole plane.

Definition of the class $\mathbb{S}_{\mathbb{F}_{t}}, C$ belongs to $\mathbb{S}_{\mathbb{F}_{t}}$ means $C$ transforms any series

$$
\sum u_{n} z^{n}=F(z),
$$

for each $z$ of $₹=\widetilde{F}(F, \&)$, into a series summable $(P t)$, to the same $(P t)$ sum. Evidently, when $\mathbb{B}=\mathbb{B}^{*}$ then $\mathbb{S}_{\mathbb{F}_{t}}$ becomes $\mathbb{F}_{t t}$, by $(\mathrm{c})$, above.

2.2. An inclusion. Denoting by

g: a simply closed rectifiable curve, situated in $\&$ and containing the origin; the limit-case $g=0$ is included; 
$J$ : a domain (open, connected) in the $y$-plane, containing the closed interval $[0,1]$,

we have the following result.

THEOREM 3. If

( I) C belongs to $\mathbb{S}_{a a}$,

(II) for each g there exists a $J$ such that $G(z, y)$ is

(a) a regular function of $y$ in $J$ for each fixed $z$ of $g$,

(b) a continuous function of both variables $(z, y)$, for $z$ on $g, y$ in $J$, and

(III) $G(z, 1)=1 /(1-z)$ for $z$ in \&,

then $C$ belongs to $\mathbb{S}_{\mathbb{G} t}$.

Corollary. Conditions (II a, b) are in particular satisfied when $G(z, y)$ is regular in (B, $[0,1])$.

Proof of the corollary. If $G(z, y)$ is regular in (\&, $[0,1])$, then in particular it is regular in the closed set $(g,[0,1])$, and consequently also in some open domain containing $(g,[0,1])$. Hence it is certainly regular in $(g, J)$ for some $J$ containing $[0,1]$, and this implies the conditions ( II a, b) of the theorem.

To prove the theorem, we first note this:

COROLlaRY 3 OF THEOREM 1. If $C$ belongs to $\mathcal{S}_{a a}, \sum_{u_{n}} z^{n}$ is analytic, and $R>0$, then the two series

$$
\begin{aligned}
& \sum_{v_{k}}(z) y^{k}, \\
& \sum_{c_{n}(y) u_{n} z^{n}}
\end{aligned}
$$

converge absolutely, uniformly, and to the same sum $f(z, y)$, for all $z$ with $|z| \leq R$, and all $y$ of a certain neighborhood of the origin, $|y| \leq \rho=\rho(R)$.

Proof. It follows from Theorem 1 that the double series

$$
\sum_{k} \sum_{n} c_{k n} u_{n} z^{n} y^{k}
$$

converges absolutely for $|z| \leq R,|y| \leq \rho(R)$, and this implies the corollary.

Proof of Theorem 3. Let $f(z)=\sum_{u_{n}} z^{n}$ [supposed continued by $\left.F(z)\right]$, and let $d$ be a closed Jordan curve around the origin, situated, together with its interior, within the star of $F(z)$. 
We obtain, for $|z| \leq R,|y| \leq \rho$ :

$$
\begin{aligned}
f(z, y) & =\sum v_{k}(z) y^{k}=\sum c_{n}(y) u_{n} z^{n} \\
& =\sum\left[c_{n}(y) z^{n} \cdot \frac{1}{2 \pi i} \int_{d} \frac{F(x)}{x^{n+1}} d x\right] \\
& =\frac{1}{2 \pi i} \cdot \sum \int_{d} c_{n}(y)\left(\frac{z}{x}\right)^{n} \cdot \frac{F(x)}{x} d x .
\end{aligned}
$$

When $x$ varies on $d, z / x$ remains bounded, say $|z / x|<R^{\prime}$. By Corollary 3 there exists a certain $\rho^{\prime}$ such that the series

$$
\sum_{c_{n}}(y)\left(\frac{z}{x}\right)^{n}
$$

and (because $F(x) / x$ is bounded on $d$ ) also the series

$$
\sum c_{n}(y)\left(\frac{z}{x}\right)^{n} \frac{F(x)}{x}
$$

are (absolutely and) uniformly convergent for all $x$ of $d$ and for $|z| \leq R,|y| \leq$ $\rho^{\prime}$.

Therefore summation and integration are permutable, and we obtain

$$
\begin{aligned}
f(z, y)=\frac{1}{2 \pi i} \int_{d} \sum_{c_{n}(y)}\left(\frac{z}{x}\right)^{n} \frac{\Gamma(x)}{x} d x \\
\quad\left[|z| \leq K,|y| \leq \rho^{\prime \prime}=\min \left(\rho, \rho^{\prime}\right)\right] .
\end{aligned}
$$

Carrying out the summation we obtain

$$
f(z, y)=\frac{1}{2 \pi i} \int_{d} g\left(\frac{z}{x}, y\right) \frac{F(x)}{x} d x \quad\left(|z| \leq R,|y| \leq \rho^{\prime \prime}\right)
$$

We need $F(z, y)$, the analytic continuation of $f(z, y)$ with regard to $y$. We first look for what is obtained by continuation of the integrand; that is to say, we consider

$$
\frac{1}{2 \pi i} \quad \int_{d} G\left(\frac{z}{x}, y\right) \frac{F(x)}{x} \quad d x=H(z, y) .
$$


We now suppose that $z$ is a fixed point of $\tilde{\xi}$, and choose the curve $d$ as follows:

As (see proof of Remark 2) $z / \odot$ is closed, bounded and situated in \&, it can be included, together with the origin, in the interior of a rectifiable curve $g$, entirely situated in (s) (the latter domain being open). In the involutory correspondence $t=z / x$ ( $z$ fixed), $g$ is the image of a rectifiable curve $d$. The segment $O z$ is in the interior, $\Theta$ on the exterior of $d$ (because $[1, \infty]$ is exterior, while the set $z / \Subset$ and the origin are interior to (8). Thus $d$ is rectifiable, includes the segment $O z$, and is situated, together with its interior, within the star of $F(z)$. When $x$ describes $d$, then $z / x=t$ describes $g$, and by hypothesis (II a, b) of the theorem there exists a $J$ such that $G(z / x, y)=G(t, y)$ is regular in $y$ and continuous in $(t, y)$ ( $t$ on $g, y$ in $J$ ). This is equivalent to:

$$
G\left(\frac{z}{x}, y\right) \text { is regular in } y \text { and continuous in }(x, y) \quad(x \text { on } d, y \text { in } J) \text {, }
$$

because $t=z / x$ is continuous in $x$ on $d$ (the origin not being on $d$ ).

As also $F(x) / x$ is continuous on $d$, it may be verified that

$$
G\left(\frac{z}{x}, y\right) \cdot \frac{F(x)}{x} \text { is regular in } y \text { and continuous in }(x, y)(x \text { on } d, y \text { in } J) \text {. }
$$

This implies the regularity of $(2.2 .4)$, that is to say:

$$
H(z, y) \text { is regular, with regard to } y \text {, in } J \text {. }
$$

On the other hand, for $|y| \leq \rho^{\prime \prime}$, we have

$$
G\left(\frac{z}{x}, y\right)=g\left(\frac{z}{x}, y\right)
$$

and from (2.2.3) and (2.2.4) it follows that

$$
f(z, y)=H(z, y)
$$

Hence for the analytic continuation we still have the identity:

$$
F(z, y)=H(z, y)=\frac{1}{2 \pi i} \int_{d} G\left(\frac{z}{x}, y\right) \frac{F(x)}{x} d x \quad(y \text { in } J),
$$

and, for each point $z$ of $₹, F(z, y)$ is regular, with regard to $y$, in $J=J(z)$. Using now the hypothesis that $J$ contains the segment $0 \leq y \leq 1$, we have thus established that 


$$
F(z, y) \text { is regular in } 0 \leq y \leq 1 \text { for each fixed } z \text { of } \mathfrak{F}
$$

To complete the proof of the theorem, we have still to verify that

$$
F(z, 1)=F(z)
$$

( $z$ point of $\mathfrak{f}$ ).

Setting $y=1$ in (2.2.8), and using the hypothesis (III), which implies

$$
G\left(\frac{z}{x}, 1\right)=\left(1-\frac{z}{x}\right)^{-1}=\frac{x}{x-z},
$$

we obtain

$$
F(z, 1)=\frac{1}{2 \pi i} \quad \int_{d} \frac{F(x)}{x-z} d x=F(z)
$$

\section{Associated Functions}

3.1. A biunique correspondence. By Theorem 1 and its Corollary 3, with each matrix of $\mathfrak{S}_{a a}$ is associated a function $G(z, y)$, which, for each given $R>0$, is regular in both variables for $|z|<R$ and $|y|<\rho$ ( $\rho$ depending on $R)$. Theorem 3 has been expressed in terms of this function.

There is a biunique correspondence between the class of functions $G(z, y)$ regular in $(0,0)$, and a certain class of matrices $C=\left(c_{k n}\right)$, containing the class $\mathbb{S}_{a a}$, and characterised by the condition

$$
\left|c_{k n}\right| \leq P^{k+1} Q^{n+1} \quad(k, n=0,1,2, \cdots)
$$

The correspondence is established by the two formulae

$$
G(z, y)=\sum \sum c_{k n} z^{n} y^{k}
$$

$$
\text { absolutely convergent for }|z|<P^{-1},|y|<Q^{-1} \text {, }
$$

$$
c_{k n}=\frac{1}{k ! n !} \frac{\partial^{k+n}}{\partial y^{k} \partial z^{n}} G(z, y)
$$

The condition of Theorem 1 may be expressed in terms of the associated function. We then obtain:

THEOREM 4. In order that $C$ should belong to $\mathbb{S}_{a a}$, it is necessary and sufficient that $G(z, y)$ be regular in $(z, 0)$ for each $z$. 
Proof. The necessity is implied by Theorem 1, Corollary 3 .

To see that the condition is sufficient, let $z$ be a fixed point of the $z$-plane. Regular in $(z, 0)$ means regular in a certain neighborhood of $(z, 0)$, say

$$
(z+\zeta, \eta)
$$$$
(|\zeta|<\rho,|\eta|<\rho)
$$

where $\rho=\rho(z)$.

We take the neighborhoods half as large, thus $\rho^{\prime}=\rho / 2$. By Borel's theorem, the closed and bounded domain $(|z|<R, y=0)$ may be covered by a finite number of the $\rho^{\prime}$ neighborhoods. Let $\left(z_{j}, 0\right),(j=1,2, \cdots, k)$, be their centers, and $\rho_{0}^{\prime}$ the smallest of their radii. An arbitrary point $(z, 0)$ of the domain $(|z| \leq$ $R, y=0)$ is situated in at least one of the covering neighborhoods, say center $z_{j}$ and radius $\rho^{\prime}=\rho^{\prime}\left(z_{j}\right)$. Hence the $\rho^{\prime}$ neighborhood of $(z, 0)$ is situated in the $2 \rho^{\prime}=\rho$ neighborhood of $(z, 0)$, in which $G(z, y)$ has been supposed regular. Consequently $G(z, y)$ is regular in

$$
\left(|z|<R+\rho_{0}, \quad|y|<\rho_{0}\right),
$$

and its Taylor series (3.1.2) is certainly absolutely convergent in

$$
\left(|z| \leq R, \quad|y| \leq \sigma<\rho_{0}\right) .
$$

From this it follows that if $\left|u_{n}\right| \leq R^{n}$, and evidently also if $\left|u_{n}\right| \leq R^{n+1}$, then $\sum u_{n}$ is transformed by $C$ into an analytic series. As $R$ has been chosen arbitrarily, the proof is complete.

3.2. A corollary. If we substitute the condition of Theorem 4 for condition (I) in Theorem 3, then this theorem is entirely expressed in terms of the associated function $G(z, y)$. Because of its importance for applications, we explicitly state the particular case of its corollary:

THEOREM 5. If

(I) $G(z, y)$ is regular

(a) in $(z, 0)$ for each $z$,

(b) in each $(z, y)$ of $(\mathbb{8}, 0 \leq y \leq 1)$, and

(II) $G(z, 1)=1(1-z)$ for each $z$ of $\&$,

then $C$ belongs to $\mathbb{S}_{\mathbb{H}}$.

3.3. Inverse considerations. The preceding two paragraphs show that it may offer some methodical advantages, especially for applications, if investigation of matrices is replaced by investigation of their associated functions. 
Inversely we may start from functions in order to construct matrices by means of formula (3.1.3); the theorems of this section may then be considered as examples showing how certain properties of the function yield corresponding properties of the associated matrix.

In view of the condition $G(z, 1)=1 /(1-z)$, a particular and most simple class of functions is obtained if we choose

$$
G(z, y)=\frac{1}{1-z h(y)},
$$

as we have but to postulate $H(1)=1$ in order to satisfy the above condition.

If $h(y)$ is supposed to be regular at the origin, then $G(z, y)$ is regular in $(0,0)$, and the associated matrix is calculated by (3.1.3); this yields

$$
c_{k n}=\frac{1}{k !} \frac{d^{k}}{d y^{k}}[h(y)]^{n} .
$$

Theorems 4 and 5, applied to this class of functions, yield the following two theorems:

ТНЕОВЕМ 6. In order that $C$ should belong to $\mathfrak{S}_{a a}$, it is necessary and sufficient that

(I) $\quad h(y)$ is regular at the origin, and

(II) $h(0)=0$.

THEOREM 7. If $h(0)=0$, then the following conditions are necessary and sufficient in order that $C$ should belong to $\mathbb{S}_{\mathbb{S}_{t}}$ :

(I) $h(y)$ is regular in $0 \leq y \leq 1$,

(II) $z h(y) \neq 1$ for each $(z, y)$ of (\&, $0 \leq y \leq 1)$, and

(III) $h(1)=1$.

REMARK. If $\&=B^{*}$, condition (II) of Theorem 7 is equivalent to $\left(\right.$ II $\left.^{*}\right) 0 \leq h(y) \leq 1$, when $0 \leq y \leq 1$.

Proof of Theorem 6. The condition of Theorem 4, namely " $G(z, y)$ is regular in $(z, 0)$ for each $z$," is equivalent to: " $h(y)$ is regular at $y=0$ and $z h(0) \neq 1$ for each $z$ ". The second part of this latter condition is further equivalent to " $h(0)=0 "$.

Proof of Theorem 7. The necessity is evident, as the conditions merely signify that $C$ behaves as a method of the respective class when applied to the 
geometric series $\sum z^{n}$. As for sufficiency, the conditions (Ia), (Ib) and (II) of Theorem 5 here are respectively equivalent to:

$$
\begin{aligned}
& h(y) \text { is regular at } y=0 \text { and } h(0)=0 ; \\
& z h(y) \neq 1 \text { for each }(z, y) \text { of }(\mathbb{\&},[0,1]) ; \\
& h(1)=1 .
\end{aligned}
$$

\section{Sequence-to-Sequence Transformations}

4.1. A sequence equivalent of Theorem 3. Theorems 1 and 3 dealt with series-to-series transformations $C$. Theorem 2 , expressed in terms of sequenceto-sequence transformations $A$, is equivalent to Theorem 1 . We now want to translate also Theorem 3 in terms of $A$.

Let $A$ be a sequence-to-sequence transformation of $\mathfrak{S}_{a a}$, and $C$ the corresponding series-to-series transformation (in the sense of $\$ 1.4$ ), so that from

$$
\sum c_{k n} u_{n}=v_{k}
$$

there follows

$$
\sum_{a_{k n}} U_{n}=V_{k}
$$

for each analytic series $\sum u_{n}$ (we always write $u_{0}+\cdots+u_{n}=U_{n}, v_{0}+\cdots+$ $\left.v_{k}=V_{k}\right)$.

Preserving the notations of (2.2), we have the following:

Definitions. The statement that $A$ belongs to $\mathcal{S}_{t t}^{\prime}$ means that $A$ transforms the sequence of partial sums of any series summable $(P t)$ into the sequence of partial sums of a series summable $(P t)$ to the same $(P t)$ sum; $A$ belongs to $\mathbb{S}^{\prime} \mathbb{F}_{t}$ means that $A$ transforms the sequence of partial sums of a series $\sum_{u_{n}} z^{n}=$ $F(z)$, for each $z$ of $\mathfrak{F}=\mathfrak{F}(F, \mathbb{B})$, into the sequence of partial sums of a series summable $(P t)$ to the same $(P t)$ sum.

Evidently " $A$ belongs to $\mathcal{F}_{t t}^{\prime}$ (or $\mathcal{S}_{\mathscr{G}}^{\prime}$ )" is equivalent to " $C$ belongs to $\mathfrak{S}_{t t}$ (or (5) $\left.{ }_{(\mathbb{H} t}\right)$ '”.

In the case of a sequence-to-sequence transformation, the associated function will be denoted by $T(z, y)$, represented, for each fixed $z$, at the origin of the $y$-plane, by

$$
T(z, y)=\sum_{t_{k}}(z) y^{k}, \text { where } t_{k}(z)=\sum_{a_{k n}} z^{n} .
$$

Using the notation $\mathbb{B}, \mathfrak{F}, g, J$ of Theorem 3 , we now may state its equivalent: 
THEOREM 8. If

(I) A belongs to $\mathbb{S}_{a a}$,

(II) for each $g$ there exists a $J$ such that $T(z, y)$ is

(a) a regular function of $y$ in $J$ for each fixed $z$ of $g$,

(b) a continuous function of both variables $(z, y)$ for $z$ on $g, y$ in $J$, and

(III) $(1-y) \cdot T(1, y)$ is

(a) regular in $0 \leq y \leq 1$, and

(b) $=1$, for $y=1$,

then $A$ belongs to $\mathbb{S}_{\mathbb{S}}^{\prime}$.

COROLLARY. The conditions (IIa,b) are satisfied in particular when $T(z, y)$ is regular at each $(z, y)$ of $(\&,[0,1])$.

Proofs. The corollary is identical to the corollary of Theorem 3, which has already been proved.

As for the theorem itself, we like to prove not only that it holds but also that it is not weaker than the corresponding Theorem 3; we shall therefore prove:

THEOREM 8a. The conditions of Theorem 8 are equivalent to those of Theorem 3.

For this purpose there must first be found a relation between $T(z, y)$ (associated with $A$ ) and $G(z, y)$ (associated with $C$ ).

Setting

$$
\begin{aligned}
& U_{n}(z)=z^{0}+z^{1}+\cdots+z^{n}=\left\{\begin{array}{cc}
\frac{1-z^{n+1}}{1-z} & \text { when } z \neq 1 \\
n+1 & \text { when } \quad z=1,
\end{array}\right. \\
& \sum_{c_{k n} z^{n}=v_{k}(z), \quad V_{k}(z)=v_{0}(z)+v_{1}(z)+\cdots+v_{k}(z),}
\end{aligned}
$$

we see that the correspondence between $A$ and $C$ implies

$$
\sum a_{k n} U_{n}(z)=V_{k}(z)
$$

Calculating the left side, we obtain

$$
\sum_{a_{k n}} U_{n}(z)=\sum a_{k n} \frac{1-z^{n+1}}{1-z}=\frac{1}{1-z}\left[\sum a_{k n}-z \sum a_{k n} z^{n}\right]
$$




$$
=\frac{1}{1-z}\left[t_{k}(1)-z \cdot t_{k}(z)\right] \text {. }
$$

Thus (4.1.2) is equivalent to

$$
\frac{1}{1-z}\left[t_{k}(1)-z t_{k}(z)\right]=V_{k}(z) \quad(z \neq 1),
$$

Substitution of these formulae in

$$
G(z, y)=\sum v_{k}(z) y^{k}=\sum\left[V_{k}(z)-V_{k-1}(z)\right] y^{k}=(1-y) \sum V_{k}(z) y^{k}
$$

yields

$$
G(z, y)=\frac{1-y}{1-z} \sum\left[t_{k}(1)-z t_{k}(z)\right] y^{k}
$$

We thus have

$$
G(z, y)=\frac{1-y}{1-z}[T(1, y)-z T(z, y)]
$$$$
(z \neq 1) \text {, }
$$

The relation wanted is $(4.1 .5)$.

To prove the equivalence of the conditions of Theorems 3 and 8 , we first observe that, by Theorem 2, the condition (I) of Theorem 8 is certainly equivalent to (I) of Theorem 3.

Further by (4.1.5) it is plain that if the conditions of Theorem 8 are satisfied, then those of Theorem 3 also are satisfied. This already establishes the truth of Theorem 8.

To complete the proof of Theorem 8a, there remains to show: If all conditions of Theorem 3 are satisfied, then conditions (II) and (III) of Theorem 8 are satisfied.

We now suppose all conditions of Theorem 3 to be satisfied; $C$ then belongs to $\mathbb{S}_{\mathbb{B}_{t}}$.

The series

$$
1+0+0+\cdots=\sum_{u_{n}} \text {, where } u_{0}=1, u_{n}=0 \quad(n \geq 1),
$$


which may be considered as the Taylor series of $F(z) \equiv 1$, is summable $(P t)$ to the sum 1 , and so must be its $C$ transform. Hence, it for this series we write

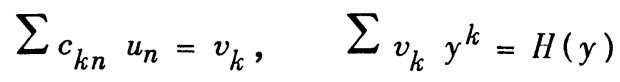

then

$$
H(y) \text { is regular in }[0,1] \text {, and } H(1)=1 \text {. }
$$

On the other hand we have

$$
\begin{aligned}
H(y) & =\sum v_{k} y^{k}=\sum\left(V_{k}-V_{k-1}\right) y^{k}=(1-y) \sum_{V_{k}} y^{k} \\
& =(1-y) \sum_{k}\left(\sum_{n} a_{k n} U_{n}\right) y^{k}=(1-y) \sum_{k}\left(\sum_{n} a_{k n}\right) y^{k} ;
\end{aligned}
$$

thus, by (4.1.1),

$$
H(y)=(1-y) T(1, y),
$$

so that (4.1.7) is exactly the condition (III) of Theorem 8.

It remains to show that conditions (II a, b) of Theorem 8 are satisfied.

It is plain that $(1-y) T(1, y)$ is still regular in an open domain $J$ containing $[0,1]$.

The conditions (II a,b) of Theorem 8 will be satisfied if $T(z, y)$ is replaced by

or by $(1-y) T(1, y)$ (as this function does not depend on $z$ ), or by $G(z, y)$ (because we then obtain (II a, b) of Theorem 3), or finally by $(1-y) T(1, y)-(1-z) G(z, y)=(1-y) z T(z, y)$ (the e-

Further from

$$
(1-z) G(z, y) \text { (as } g \text { does not pass through } z=1 \text { ), }
$$

$$
\text { quality following from (4.1.5)). }
$$

$$
\begin{aligned}
& G(z, y)=1 /(1-z) \quad \text { when } y=1, \\
& (1-y) T(1, y)=1 \quad \text { when } y=1,
\end{aligned}
$$

it follows that

$$
(1-y) z T(z, y)=0 \text { when } y=1 \text {. }
$$

Hence $z T(z, y)$ is regular in $y$ wherever $(1-y) z T(z, y)$ is regular in $y$, and the conditions (II a, b) will be satisfied, if $T(z, y)$ is replaced by $z T(z, y)$. 
Now, for $z \neq 0, T(z, y)$ is regular in $y$ wherever $z T(z, y)$ is regular in $y$. Consequently the conditions (II a,b) of Theorem 8 are certainly satisfied when $z \neq 0$.

For $z=0$ we may calculate $T(0, y)$ in the same way as we $\operatorname{did}$ for $z=1$. We start from the series

$$
1-1+0+0+\cdots
$$

which is the Taylor series of $F(z)=1-z$ for $z=1$, and certainly summable $(P t)$.

Its $C$ transform $\sum v_{k}$, where

$$
v_{k}=\sum c_{k n} u_{n}\left(=c_{k 0}-c_{k_{1}}\right),
$$

must also be summable $(P t)$; that is to say, if we write $\sum_{v_{k}} y^{k}=P(y)$, then

$$
P(y) \text { is regular in }[0,1] \text {, and } P(1)=0 \text {. }
$$

On the other hand we obtain

$$
P(y)=(1-y) \sum\left(\sum a_{k n} U_{n}\right) \text {, where now } U_{0}=1, U_{n}=0 \quad(n \geq 1) \text {. }
$$

Thus, by (4.1.1),

$$
P(y)=(1-y) T(0, y) \text {, }
$$

so that $T(0, y)$ is regular in $0 \leq y \leq 1$, and consequently also in an open domain $J$ containing $[0,1]$. This signifies that the conditions ( $\mathrm{II} \mathrm{a}, \mathrm{b})$ of Theorem 8 are also satisfied when $z=0$, and completes the proof that all conditions of Theorem 8 are implied by those of Theorem 3 .

4.2. Sequence equivalents of other results. Finally we state the equivalents of the formulae and theorems of $\$ 3$.

The biunique correspondence between the matrices $A$ of $\mathbb{S}_{a a}$ and the class of associated functions $T(z, y)$ is given by the two formulae:

$$
t(z, y)=\sum \sum a_{k n} z^{n} y^{k}
$$

and

$$
a_{k n}=\frac{1}{k ! n !} \frac{\partial^{k+n}}{\partial y^{k} z^{n}} T(z, y) \quad(z=0, y=0) .
$$


$T(z, y)$ is the analytic continuation of $t(z, y)$ with respect to $y$, for each fixed $z$.

If $A=\left(a_{k n}\right)$ belongs to $\mathbb{S}_{a a}$, then for each $R>0$ the double series is absolutely convergent for

$$
|z|<R, \quad|y|<\rho=\rho(R) .
$$

In terms of $T(z, y)$, Theorem 2 and Theorem 8, Corollary, respectively yield the following two theorems.

THEOREM 9. In order that $A=\left(a_{k n}\right)$ of (4.2.2) should belong to $\mathbb{S}_{a a}$, it is necessary and sufficient that $T(z, y)$, considered as a function of both variables, be regular at $(z, 0)$ for each $z$.

THEOREM 10. If

(I) $T(z, y)$, as a function of two variables, is regular

(a) in $(z, 0)$ for each $z$, and

(b) in each $(z, y)$ of (\&, $[0,1])$, and

(II) $(1-y) T(1, y)$, as function of $y$, is

(a) regular in $0 \leq y \leq 1$, and

(b) $=1$ when $y=1$,

then $A$ belongs to $\mathbb{S}_{\text {(S) }}^{\prime}$.

A very particular and simple class of functions $T(z, y)$, satisfying the conditions of Theorem 8, may be obtained as follows:

Condition ( III b), postulating

$$
(1-y) T(1, y)=1 \text { when } y=1 \text {, }
$$

is satisfied when we place

$$
(1-y) T(1, y) \equiv 1
$$

thus

$$
T(1, y)=\frac{1}{1-y} .
$$

Among the functions $T(z, y)$ satisfying (4.2.3), we may choose the special class

$$
T(z, y)=\frac{1}{1-y h(z)},
$$


where we have only to postulate $h(1)=1$ in order to obtain (4.2.3).

If $h(z)$ is regular at the origin, then $T(z, y)$ is regular at $(0,0)$ and formula (4.2.2) gives the associated matrix $A$.

As for $(z, y)=(0,0)$, we have here

$$
\frac{1}{k ! n !} \frac{\partial^{k+n}}{\partial y^{k} \partial z^{n}} \quad T(z, y)=\frac{1}{n !} \frac{d^{n}}{d z^{n}} \quad[h(z)]^{k},
$$

and the matrix $A$ is determined by

$$
a_{k n}=\frac{1}{n !} \frac{d^{n}}{d z^{n}}[h(z)]^{k} .
$$

The possibility of associating a matrix $A$ with a function $h(z)$ by formula (4.2.5) was pointed out by J. Sonnenschein [1], who gave the conditions

$$
h(1)=1 \text {, and } h(z) \neq 1 \text { when } z \neq 1 \text {, }
$$

in order that $A$ be consistent with analytic continuation, when applied to the sequence of partial sums of the geometric series $\sum z^{n}$ and its finite linear combinations

$$
\lambda_{1} \sum z_{1}^{n}+\lambda_{2} \sum z_{2}^{n}+\cdots+\lambda_{p} \sum_{z_{p}^{n}},
$$

thus to series $\sum_{u_{n}}$, where

$$
u_{n}=\lambda_{1} z_{1}^{n}+\cdots+\lambda_{p} z_{p}^{n} \quad(n=0,1, \cdots)
$$

In view of (4.2.4) we may easily verify the following two theorems, as immediate applications of Theorems 9 and 10 respectively.

TheOrem 11. If $h(z)$ is an integral function, then $A$ belongs to $\mathbb{S}_{a a}$.

THEOREM 12. If

(I) $\quad h(z)$ is an integral function,

(II) $h(z)$ is not in $[1, \infty]$, when $z$ in अ, and

(III) $h(1)=1$,

then A belongs to $\mathbb{C}_{\vec{A}}$.

Theorem 12a. In Theorem 12 the conditions (II) and (III) are necessary. 
Proof of Theorem 11. Evidently if $h(z)$ is regular in the whole plane then, by (4.2.4), $T(z, y)$ is regular when $1-y h(z) \neq 0$. Now let $z$ be a fixed point. As $h(z)$ is bounded in any bounded region, we have

$|y h(z)|<1$; consequently $1-y h(z) \neq 0$ in a certain neighborhood of $(z, 0)$.

Proof of Theorem 12. Condition (II) yields

$$
y h(z) \neq 1 \quad(0 \leq y \leq 1, z \text { in (8) }),
$$

which implies condition (Ib) of Theorem 10. Further $h(1)=1$ yields $(1-y) \times$ $T(1, y) \equiv 1$, which implies (II a, b) of Theorem 10 .

Proof of Theorem 12a. For the necessity of condition (III) evidence is obtained by application of $A$ to the sequence $1,1,1, \ldots$, which is the sequence of partial sums of $1+0+0+\cdots$. The necessity of condition (II) is verified by application of $A$ to the sequence of partial sums of the geometric series $\sum_{z^{n}}$, thus to the sequence

$$
\frac{1-z^{n+1}}{1-z}=\frac{1}{1-z}\left[1-z^{n+1}\right]=a+b z^{n} \quad(z \neq 1)
$$

\section{SOME REMARKS}

5.1. For applications, the class $\mathfrak{S}_{t t}=\mathbb{S}_{\mathbb{B} *_{t}}$ may be of more importance than any other $\mathcal{S}_{\mathbb{F}_{t}}$. Theorems on the particular case are obtained by replacing $\&$ by \&* $^{*}$ in Theorems $3,5,7,8,10$, and 12 . See also the remark following Theorem 7.

5.2. This paper is not concerned with summability. However we point out that replacement of the domain $J$ by an open circle $|y|\langle r$ with $r>1$ in conditions and proof of Theorem 3, readily yields:

If in the conditions of either of the theorems mentioned in (5.1) the domains $[0,1]$ and $J$ (containing $[0,1]$ ) are replaced by

$$
|y| \leq 1 \text { and }|y|<r
$$

respectively, then a transformation ( $C$ or $A$ ) satisfying the new conditions of the theorem certainly sums $\sum u_{n} z^{n}$ to the sum of analytic continuation, for each $z$ of $₹=\{(F, \mathbb{F})$. In Theorem 12, " $h(z)$ is not in $[1, \infty]$ " then is to be replaced by " $|h(z)|<1$ " (compare proof of the same theorem). 
5.3. Theorem 1 characterizes the linear operations defined at any point of the space of all analytic sequences, and can also be proved by the methods of operator theory; however it takes some pages to establish the basic properties of this space, after introduction of an appropriate metric (for instance $|x|=$ $\sup \left|x_{n}\right|^{1 / n}$ or an only locally defined distance $|x-y|=\left[\sum\left(x_{n}-y_{n}\right)^{2}\right]^{1 / 2}$. or the corresponding hermitian expression).

\section{REFERENCE}

1. Jakob Sonnenschein, Sur les séries divergentes, Acad. Roy. Belgique. Bull. Cl. Sci. (5) 35 (1949), 594-601.

George Washington University 



\section{PACIFIC JOURNAL OF MATHEMATICS}

\section{EDITORS}

R. M. RoBINSON

University of California Berkeley 4, California

\section{*R. P. Dilworth}

Califomia Institute of Technology Pasadena 4, California

\section{E. F. BECKENBACH, Managing Editor}

University of California

Los Angeles 24, California

*During the absence of Herbert Busemann in 1952.

\section{ASSOCIATE EDITORS}

\author{
R. P. DILWORTH \\ HERBERT FEDERER \\ MARSHALL HALL
}

\author{
P. R. HALMOS \\ HEINZ HOPF
}

R. D. JAMES

\author{
BØRGE JESSEN \\ PAUL LÉVY \\ GEORGE PÓLYA
}

\author{
J. J. STOKER \\ E. G. STRAUS
}

KÖSAKU YOSIDA

\section{SPONSORS}

UNIVERSITY OF BRITISH COLUMBIA

CALIFORNIA INSTITUTE OF TECHNOLOGY

UNIVERSITY OF CALIFORNIA, BERKELEY

UNIVERSITY OF CALIFORNIA, DAVIS

UNIVERSITY OF CALIFORNIA, LOS ANGELES

UNIVERSITY OF CALIFORNIA, SANTA BARBARA

OREGON STATE COLLEGE

UNIVERSITY OF OREGON

\author{
UNIVERSITY OF SOUTHERN CALIFORNIA \\ STANFORD UNIVERSITY \\ WASHINGTON STATE COLLEGE \\ UNIVERSITY OF WASHINGTON \\ AMERICAN MATHEMATICAL SOCIETY \\ NATIONAL BUREAU OF STANDARDS, \\ INSTITUTE FOR NUMERICAL ANALYSIS
}

Mathematical papers intended for publication in the Pacific Journal of Miathematics should be typewritten (double spaced), and the author should keep a complete copy. Manuscripts may be sent to any of the editors. All other communications to the editors should be addressed to the managing editor, E. F. Beckenbach, at the address given above.

Authors are entitled to receive 100 free reprints of their published papers and may obtain additional copies at cost.

The Pacific Journal of Mathematics is published quarterly, in March, June, September, and December, by the University of California, Berkeley 4, California. The price per volume (4 numbers) is $\$ 8.00$; single issues, $\$ 2.50$. Special price to individual faculty members of supporting institutions and to individual members of the American Mathematical Society: $\$ 4.00$ per volume; single issues, $\$ 1.25$.

Subscriptions, orders for back numbers, and changes of address should be sent to the publishers, University of California Press, Berkeley 4, California.

Printed at Ann Arbor, Michigan. Entered as second class matter at the Post Office, Berkeley, California.

\section{UNIVERSITY OF CALIFORNIA PRESS • BERKELEY AND LOS ANGELES}




\section{Pacific Journal of Mathematics}

\section{Vol. 2, No. $2 \quad$ February, 1952}

L. Carlitz, Some theorems on Bernoulli numbers of higher order...

Watson Bryan Fulks, On the boundary values of solutions of the heat equation........................................ 141

John W. Green, On the level surfaces of potentials of masses with fixed center of gravity................................... 147

Isidore Heller, Contributions to the theory of divergent series .......... 153

Melvin Henriksen, On the ideal structure of the ring of entire functions . . . 179

James Richard Jackson, Some theorems concerning absolute neighborhood retracts........................................ 185

Everett H. Larguier, Homology bases with applications to local connectedness ................................... 191

Janet McDonald, Davis's canonical pencils of lines ................ 209

J. D. Niblett, Some hypergeometric identities . . . . . . . . . . . . . . . . . . 219

Elmer Edwin Osborne, On matrices having the same characteristic equation...................................... 227

Robert Steinberg and Raymond Moos Redheffer, Analytic proof of the

Lindemann theorem ................................. 231

Edward Silverman, Set functions associated with Lebesgue area ......... 243

James G. Wendel, Left centralizers and isomorphisms of group algebras . . 251

Kosaku Yosida, On Brownian motion in a homogeneous Riemannian space. 